\title{
554 THE PANORAMA OF TUMOR INTRINSIC IMMUNE REGULATORS EXHIBITED BY GENOME-WIDE CRPISR IMMUNE SCREEN INTEGRATED WITH COMPREHENSIVE CLINICAL DATASET ANALYSIS
}

${ }^{1}$ Jiakai Hou*, ${ }^{2}$ Yunfei Wang, ${ }^{3}$ Leilei Shi, ${ }^{3}$ Yuan Chen, ${ }^{1}$ Chunyu Xu, ${ }^{1}$ Arash Saeedi, ${ }^{3}$ Ke Pan, ${ }^{1}$ Ritu Bohat, ${ }^{1}$ Nicholas Egan, ${ }^{3}$ Jodi McKenzie, ${ }^{3}$ Rina Mbofung, ${ }^{3}$ Leila Williams, ${ }^{3}$ Zhenhuang Yang, ${ }^{3}$ Ming Sun, ${ }^{1}$ Xiaofang Liang, ${ }^{3}$ Jordi Rodon Ahnert, ${ }^{1}$ Navin Varadarajan, ${ }^{3}$ Cassian Yee, ${ }^{3}$ Yiwen Chen, ${ }^{2}$ Patrick Hwu, 'Weiyi Peng. ${ }^{1}$ University of Houston, Houston, TX, USA; ${ }^{2}$ H. Lee Moffitt Cancer Center, Tampa, FL, USA; ${ }^{3}$ MD Anderson Cancer Center, Houston, TX, USA

Background Despite approval of immunotherapy for wide ranges of cancers, the majority of patients fail to respond to immunotherapy or relapse following initial response which is partially attributed to immunosuppression co-opted by tumor cells. However, it is challenging to utilize conventional methods to systematically evaluate the potential of tumor intrinsic factors to act as immune regulators in cancer patients.

Methods In this study, an unbiased integrative strategy were designed to leverage the complementary strength of in vitro functional genomic screens and multi-omics clinical dataset to assess roles of individual tumor-intrinsic factors in regulating $\mathrm{T}$ cell tumor infiltration and $\mathrm{T}$ cell-mediated tumor killing, the two most important rate-limiting steps of cancer immunotherapy. Initially, a genome-wide CRISPR-Cas9 screening system using paired murine tumors and tumor-reactive $\mathrm{T}$ cells was employed to globally screen tumor intrinsic factors modulating the tumor sensitivity to $\mathrm{T}$ cell-mediated killing. Then, findings from the screening were integrated with the bioinformatics analysis of clinical datasets to further evaluate roles of each tumor intrinsic factor in governing antitumor immunity.

Results The integrative analysis successfully identified several novel tumor intrinsic factors as effectors of immune resistance, but also demonstrated distinct roles of these factors in controlling immune cell trafficking and tumor sensitivity to T cellmediated killing. Among these factors, candidates controlling both rate-limiting steps of $\mathrm{T}$ cell tumor infiltration and $\mathrm{T}$ cellmediated tumor killing were termed as "Dual immune resistance regulators" and the remaining factors whose expression levels were not associated with tumor immune infiltration were termed as "Cytotoxicity resistance regulators". By selecting PRMT1 and RIPK1 as the representatives of these two groups respectively, we confirmed that genetically depletion of PRMT1 and RIPK1 sensitized tumors to T-cell mediated killing via two independent experimental approaches. Furthermore, inhibiting Prmt1 or Ripk1 sensitizes tumors to cancer immunotherapy, such as anti-PD-1 and anti-OX40 treatments (Tumor size $(\mathrm{mm} 2)$ on day 21 after tumor inoculation: for anti-PD-1 treatment, Ctrl 84.05 \pm 23.10, PRMT1 KO 7.30 \pm 7.81 , RIPK1 KO 2.03 \pm 4.96 ; for anti-OX40 treatment, Ctrl

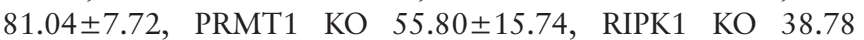
\pm 14.06 ) and extended the survival of tumor-bearing mice. Moreover, by using a RIPK1-specific inhibitor, GSK2982772, we demonstrated that targeting cytotoxicity resistance regulators could enhance the antitumor activity of $\mathrm{T}$ cell-based cancer immunotherapy, despite limited impact on $\mathrm{T}$ cell tumor infiltration.

Conclusions Collectively, our data not only demonstrate distinct immunoregulatory roles and therapeutic potentials of PRMT1 and RIPK1 in T cell-mediated antitumor activity, but also provide a rich resource of novel targets for rational immuno-oncology combinations. 